\title{
Review
}

\section{Small substrate, big surprise: fold, function and phylogeny of dihydroxyacetone kinases}

\author{
B. Erni*, C. Siebold ${ }^{+}$, S. Christen, A. Srinivas, A. Oberholzer ${ }^{++}$and U. Baumann \\ Department of Chemistry and Biochemistry, University of Bern, Freiestr. 3, 3012 Bern (Switzerland), \\ e-mail: bernhard.erni@ibc.unibe.ch
}

Received 27 October 2005; received after revision 21 December 2005; accepted 3 January 2006

Online First 28 February 2006

\begin{abstract}
Dihydroxyacetone (Dha) kinases are a family of sequence-conserved enzymes which utilize either ATP (in animals, plants and eubacteria) or phosphoenolpyruvate (PEP, in eubacteria) as their source of high-energy phosphate. The kinases consist of two domains/subunits: DhaK, which binds Dha covalently in hemiaminal linkage to the $\mathrm{N} \varepsilon 2$ of a histidine, and DhaL, an eight-helix barrel that contains the nucleotide-binding site. The PEPdependent kinases comprise a third subunit, DhaM, which rephosphorylates in situ the firmly bound ADP cofactor. DhaM serves as the shuttle for the transfer of phosphate from the bacterial PEP: carbohydrate phosphotransferase system (PTS) to the Dha kinase. The DhaL
\end{abstract}

and DhaK subunits of the PEP-dependent Escherichia coli kinase act as coactivator and corepressor of DhaR, a transcription factor from the $\mathrm{AAA}^{+}$family of enhancerbinding proteins. In Gram-positive bacteria genes for homologs of DhaK and DhaL occur in operons for putative transcription factors of the TetR and DeoR families. Proteins with the Dha kinase fold can be classified into three families according to phylogeny and function: Dha kinases, DhaK and DhaL homologs (paralogs) associated with putative transcription regulators of the TetR and DeoR families, and proteins with a circularly permuted domain order that belong to the DegV family.

Keywords. AAA ${ }^{+}$-ATPase, ADP, coenzyme, glycerone, hemiaminal, phosphoenolpyruvate, phosphotransferase.

\section{Dihydroxyacetone: the substrate}

Dihydroxyacetone (Dha) is one of the simplest carbohydrates. It consists of only three carbon atoms, is non-chiral and in principle can be formed under prebiotic conditions by spontaneous condensation of three molecules of formaldehyde. Glyceraldehyde can be formed by tautomerization (hydrogen shift) of Dha, and the two trioses then can combine by aldol condensation to form sorbose

* Corresponding author.

+ Present address: Division of Structural Biology, The Henry Wellcome Building for Genomic Medicine, The University of Oxford, Roosevelt Drive, Oxford OX3 7BN (United Kingdom).

${ }^{++}$Present address: Section of Structural Biology, The Institute of Cancer Research 237 Fulham Road, London SW3 6JB (United Kingdom). and fructose $[1,2]$. The two hexoses (or hexose phosphates under biotic conditions) can serve as precursors for nearly every sugar that exists in nature (for the structural formulas of Dha and its reaction partners see Fig. 1). Solid dihydroxyacetone $\left(\mathrm{C}_{3} \mathrm{H}_{6} \mathrm{O}_{3}\right.$, glycerone $)$ exists as a dimeric hemiketal that in aqueous solution dissociates into a 4:1 mixture of monomeric ketone and ketone hydrate (gem-diol) [3]. Dha is chemically reactive like other ketose sugars but because of its small size cannot react intramolecularly to form cyclic hemiketals. Instead, it reacts intermolecularly with proteins and nucleic acids by Maillardtype cross-linking reactions $[4,5]$. Owing to its chemical reactivity Dha is an important precursor of building blocks for use in organic synthesis [2] and has many technical applications. Dha is the active ingredient of sunless tanning (self-tanning) sprays and lotions [6, 7], and 6-acetyl- 


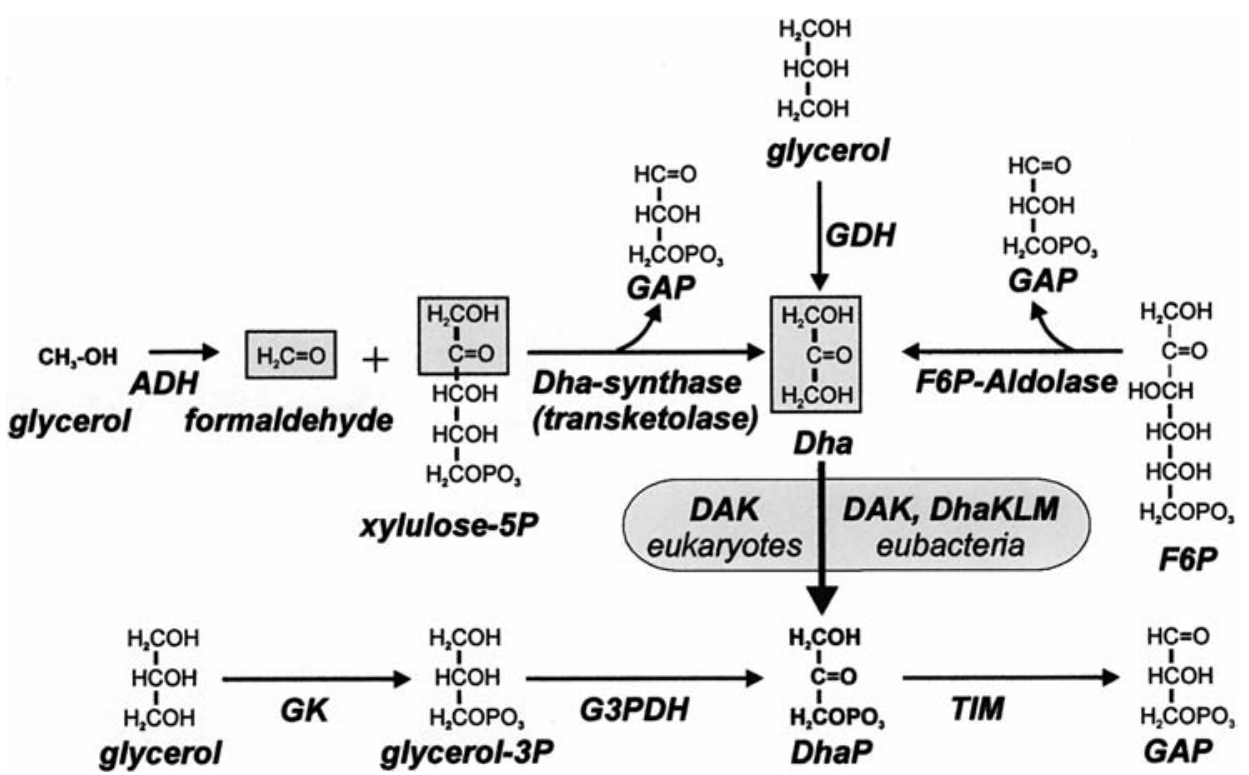

Figure 1. Metabolic origins of Dha. Alcohol dehydrogenase (ADH) and Dha-synthase are used by methlylotrophic yeast for the assimilation of methanol. Membrane-bound and soluble glycerol dehydrogenases (GDH) occur in bacteria. Aldolases are ubiquitous. The Dha kinases (DAK and DhaKLM) also phosphorylate D-glyceraldehyde and possibly other short-chain ketoses and aldoses (not shown). Glycerol kinase (GK) and glycerol-3-phosphate deyhdrogenase $(\mathrm{G} 3 \mathrm{PDH})$ are an alternative for the conversion of glycerol to dihydroxyacetone phosphate(DhaP). Triosephosphate isomerase (TIM) interconverts DhaP and glyceraldehyde-3-phosphate (GAP). The rectangular boxes highlight the carbon bond-forming reactions of Dha.

1,2,3,4-tetrahydropyridine, the product of the 'Hunter reaction' between Dha and L-proline, contributes to the roasty odour of crackers, popcorn and bread crust $[8,9]$.

Dha is utilized as a carbon source by bacteria and yeasts, and there are several pathways by which free Dha is formed in living cells (Fig. 1). (i) The yeast Schizosaccharomyces pombe and the bacteria Klebsiella pneumoniae and Citrobacter freundii oxidize glycerol with a soluble $\mathrm{NAD}^{+}$-dependent glycerol dehydrogenase under anaerobic conditions, and Dha is then phosphorylated by a Dha kinase [10-13]. (ii) Gluconobacter oxydans and related bacteria oxidize glycerol with a membrane-bound, pyrroloquinoline quinone (PQQ)-dependent glycerol dehydrogenase that is connected via ubiquinone to a respiratory chain. The oxidation reaction takes place on the outer face of the membrane, and Dha is released into the medium [14]. Industrial Gluconobacter strains yield up to $220 \mathrm{~kg} / \mathrm{m}^{3}$ of Dha because the dehydrogenase retains activity even after irreversible growth inhibition of cells by Dha [15]. (iii) Methylotrophic yeasts produce Dha during methanol assimilation. In this pathway, methanol is first oxidized to formaldehyde. To this one-carbon compound a two-carbon hydroxyethyl group is then transferred from xylulose-5-phosphate by Dha-synthase, a thiamine pyrophosphate-dependent transketolase [1621]. Bacteria, for comparison, utilize other routes to assimilate formaldehyde and its oxidation product $\mathrm{CO}_{2}$, namely the ribulose monophosphate (RuMP) cycle, the serine pathway and the reductive pentose phosphate cycle $[21,22]$. None of them produces Dha. Only recently have the xylulose monophosphate cycle enzymes also been discovered in a methanol-utilizing carboxydobacterium $[23,24]$.

Little is known about the metabolic origin and the fate of free Dha in higher eukaryotes and plants. Dha and glyceraldehyde may be formed by slow aldol cleavage of ketopentoses and ketohexoses or ketose monophosphates $[25,26]$ and by the oxidation of glycerol by $\mathrm{NAD}^{+}$-dependent alcohol dehydrogenases and NADPH-dependent aldehyde reductases [27]. Dha stimulates the secretion of insulin [28] and is utilized as gluconeogenic precursor by mammalian tissues [28-32]. Dha and pyruvate have been claimed to increase muscle mass and aerobic endurance capacity $[33,34]$, and Dha thus made its way also into over-the-counter weight-loss products.

\section{Occurrence and biochemistry of Dha kinases}

Dha kinases occur in eubacteria, plants and animals, including humans. There exist two different enzymes with Dha kinase activity: (i) In the narrow sense, Dha kinases that phosphorylate Dha and D-glyceraldehyde (EC 2.7.1.28 and EC 2.7.1.29). Such kinases consist of a Dha binding $\alpha / \beta$ domain and a nucleotide-binding all $\alpha$-helical domain. They are the topic of this review. (ii) Glycerol kinases (EC 2.7.1.30) that consist of two structurally homologous $\alpha / \beta$ domains. They have the binding sites for glycerol and ATP in the cleft between the two domain [35, 36]. Glycerol kinases are less specific, and in addition to 
glycerol also phosphorylate Dha and glyceraldehyde [3740]. The Dha-specific kinases have been characterized in bacteria $[10,41]$ and in methylotrophic yeast (for a review see [42-44]). They have been purified from yeasts $[16,17,42,43,45-49]$, algae [50], tomato [51], rat brain [31], porcine kidney [52] and from bacteria, viz. C. freundii [10, 41], K. pneumoniae [13] and E. coli [53].

Dha kinases can be grouped into two structurally related families (Fig. 2) according to their source of high-energy phosphate, namely ATP and phosphoenolpyruvate (PEP). The ATP-dependent kinases occur in yeasts, animals, plants and eubacteria, the PEP-dependent only in eubacteria. The existence of a PEP-dependent kinase was first noticed by Jin and Lin [54] in an E. coli double mutant lacking the ATP-dependent glycerol kinase and the $\mathrm{NAD}^{+}$-dependent glycerol-phosphate dehydrogenase. While looking for an ATP-dependent Dha kinase, they

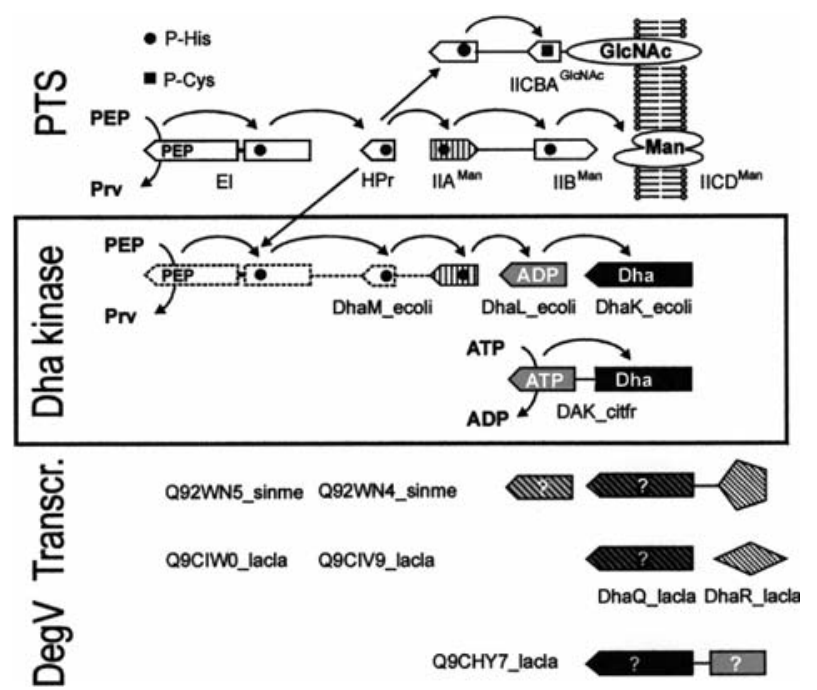

Figure 2. Dha kinases and the bacterial phosphoenolpyruvate:sugar phosphotransferase system (PTS). Center: a PEP-dependent heterotrimeric Dha kinase (DhaK, DhaL, DhaM), and an ATP-dependent two-domain Dha kinase. Top: Domain structure of the PTS. Shown are the core components of the E. coli PTS (EI and HPr) with two transporters for mannose (IIAB ${ }^{\text {Man }}$, IIC Ian $^{\text {, IID }}{ }^{\text {Man }}$ ) and GlcNAc (IICBA ${ }^{\mathrm{GlcNAc}}$ ). Bottom: Transcription regulators from $S$. meliloti consisting of a SorC-DhaK fusion protein and a DhaL-like subunit. Transcription regulators from L. lactis: DhaQ is a DhaK paralog; DhaR belongs to the TetR-family of transcription factors. Domain structure of the DegV protein YfhG (Q9CHY7) of L. lactis. DNA-binding domains/subunits are shown diagonally striped. Domains homologous to EI and HPr (white), IIA (vertically striped), DhaL (grey) and DhaK (black) are vertically aligned.

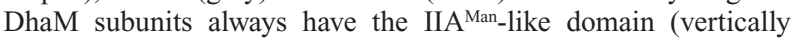
striped) and, depending on the strain, between one and three $\mathrm{HPr}$ and EI-like phosphotransferase domains (white). DhaM of E. coli has three domains (IIA ${ }^{\text {Man }}$, EI, and HPr-like). Active site His and Cys are indicated as circles and squares. Arrows indicate the phosphoryl flow from the core PTS proteins (EI and HPr), to the the PEP-dependent carbohydrate transporters and the PEP-dependent Dha kinases. The '?' indicates that the ligands are not known. Proteins of unknown function are identified by their Swiss-Prot accession numbers. observed that an E. coli ptsI mutant, which lacked EI of the phosphoenolpyruvate:sugar phosphotransferase system (PTS; Fig. 2) and therefore did not grow on PTS sugars also no longer grew on Dha (for reviews on the PTS see [55-57]). They subsequently isolated a transposon mutant unable to grow on Dha but that grew normally on PTS sugars. The transposon-tagged gene was mapped to 26 min in the E. coli chromosome, termed pts $D$ and proposed to encode a Dha-specific enzyme II of the PTS [58]. However, this putatively membrane-bound PtsD activity has never been confirmed biochemically. Instead, the operon at $26 \mathrm{~min}$ on the E. coli chromosome was found to encode two proteins (DhaK and DhaL) that are homologous to ATP-dependent Dha kinases, and one multidomain protein (DhaM) that is homologous to enzyme I and HPr, two phosphotransfer proteins of the PEP:carbohydrate (PTS) [59]. The DhaK and DhaL subunits were also found as upregulated protein spots in the proteome of an E. coli ptsI (EI) mutant [60]. Both findings confirmed that Jin and Lin indeed discovered a Dha kinase and that this kinase was functionally and genetically associated with the PTS.

ATP-dependent Dha kinases are single polypeptide twodomain proteins, while the PEP-dependent forms consist of three subunits, DhaK, DhaL and DhaM (Fig. 2). DhaK and $\mathrm{DhaL}$ are homologous to the amino terminal $\mathrm{K}$ - and the carboxy terminal L-domain of the ATP-dependent kinases. DhaK contains the binding site for Dha, and DhaL contains the nucleotide-binding site. DhaM is a phosphohistidine protein that transfers phosphoryl groups from a phosphoryl carrier protein of the PTS (HPr or EI) to the DhaL-ADP complex [53, 61, 62].

\section{Structure and molecular function of Dha kinases}

The structures of the ATP-dependent $C$. freundii Dha kinase (DAK) and of the homologous DhaK subunit of the E. coli kinase have been solved by X-ray crystallography $[62,63]$. The two kinases assume the same fold but differ in details (Fig. 3). The structure of the $C$. freundii DAK will serve as a frame of reference for the following discussion. This kinase is a homodimer of two intertwined subunits. Each subunit consists of two domains ( $\mathrm{K}$ and L), which are connected by a 20-residue-long, extended linker. The domains are swapped such that the amino terminal K-domain of one subunit is complexed with the carboxy terminal L-domain of the other (Fig. 3a).

The amino terminal K-domain consists of two six-stranded $\alpha / \beta$ folds that together form a three-layered mixed $\alpha / \beta$ sandwich (Figs. 3a, c). The core of the first fold consists of a four-stranded open pleated sheet with the same topology as the IIA subunit of the mannose family of the PTS transporters $[64,65]$. The core of the second fold has a topology similar to the carboxy terminal domain of the 


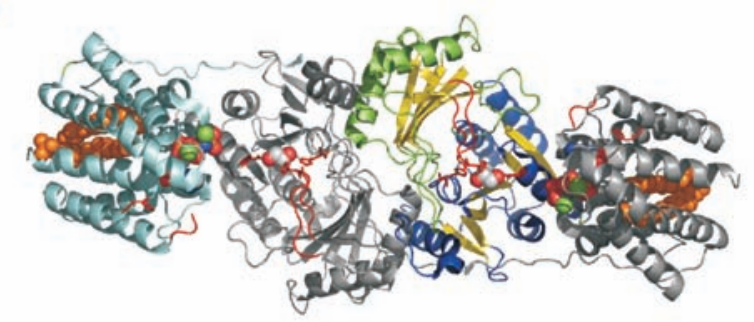

b
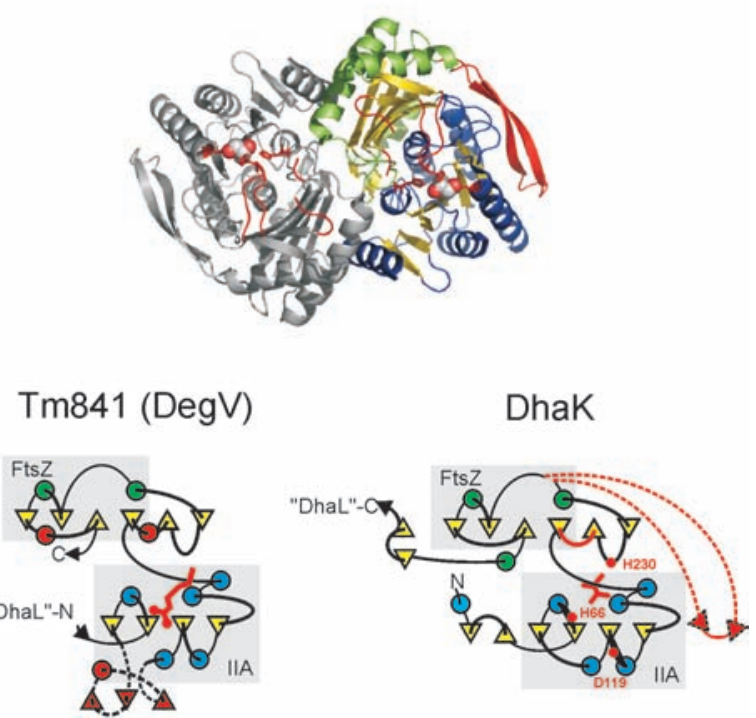

Figure 3. Structure of the Dha kinase from $C$. freundii $(a)$ and E. coli $(b)$. Cartoon representation of the Dha kinase dimers. The subunits of the dimers are coloured in blue/green and grey and the DhaL domains in cyan. The $\alpha$-helices of the first and second DhaK subdomain are coloured in blue and green, respectively, $\beta$-sheets in yellow. The active site His (red sticks) of DhaK with Dha (spheres) are shown. The nucleotide (AMPPNP, grey), the two $\mathrm{Mg}^{2+}$ ions (green) bound to the DhaL-domain, and the phosphatidic acid molecule which occupies the interior of helix-barrel (orange) are shown as spheres. The $\beta$-hairpin of $E$. coli $\mathrm{DhaK}$ is shown in red. A loop which is ordered in the $C$. freundii DhaK domain and disordered in the $E$. coli DhaK subunit is shown in red. (c) Topological diagrams of the Dha-binding DhaK subunit and of the fatty-acid binding TM841 subunit are coloured as in $a$ and $b$. Circles represent $\alpha$-helices, triangles $\beta$-strands pointing away (up) and towards (down) the viewer. The $\beta$-hairpin in $E$. coli $\mathrm{DhaK}$ and extra secondary structure elements of TM841 are shown in red. The IIA and FtsZ core folds are boxed. PDB accession codes: C. freundii 1UN9; E. coli, 1OI2; TM841, 1MGP.

cell division protein FtsZ. The K-domain provides the dimerization interface consisting of two pairs of helices. The substrate, Dha, is bound covalently in hemiaminal linkage to the K-domain (Fig. 4a). The C-N bond is formed between the carbonyl carbon of Dha and the imidazole ring $(\mathrm{N} \varepsilon 2)$ of His-220, and the hemiaminal is stabilized by a hydrogen bond between the geminal hydroxyl group and $\mathrm{N} \varepsilon 2$ of His-61. Dha is oriented by additional hydrogen bonds extending from the $\mathrm{C} 1$ - and $\mathrm{C} 3$-hydroxyl groups of Dha to the $\gamma$-carboxyl group of Asp-114 and to the main chain amide of Gly-59. There is no evidence for a role of the hemiaminal in covalent catalysis [66]. Rather, hemiaminal formation is a 'clever strategy' to discriminate between a short-chain carbonyl compound (Dha or glyceraldehyde) that can form a hemiaminal and the isosteric alcohol (glycerol) that cannot form such a covalent bond. The E. coli Dha kinase, in fact, is not inhibited even by as high as $2 \mathrm{M}$ glycerol [66].

The carboxy terminal L-domain of the $C$. freundii DAK (Fig. 3a) consists of an eight-stranded $\alpha$-helical barrel of regular up-down topology [62] with the nucleotide-binding site in a shallow depression at the narrow end of the barrel. This fold is novel and unlike any other kinase with known structure (for reviews see [67, 68]). The hydrophobic interior of the barrel is filled with a phospholipid molecule which was retained during all purification steps. The lipid headgroup protrudes from the wider end of the barrel, where it is accessible to phospholipase D, and the lipid can be removed with a mild detergent. Detergent-washed Dha kinase remains active, suggesting that the lipid plays no role in catalysis but may stabilize the structure of the barrel. The four helix-connecting loops at the narrow end of the barrel form the side wall of the ATP-binding site. The first and second loops embrace the phosphate groups of ATP, the third contacts the adenine base. The fourth loop, which is disordered in the $C$. freundii structure, most likely forms a flexible cap over the binding site. The three phosphate groups of ATP are coordinated by two $\mathrm{Mg}^{2+}$ ions, which in turn are complexed by three $\gamma$-carboxylates from aspartates in the first loop (Fig. 4b). This novel ATP-binding site differs in two aspects from those of other nucleotide-binding proteins

a
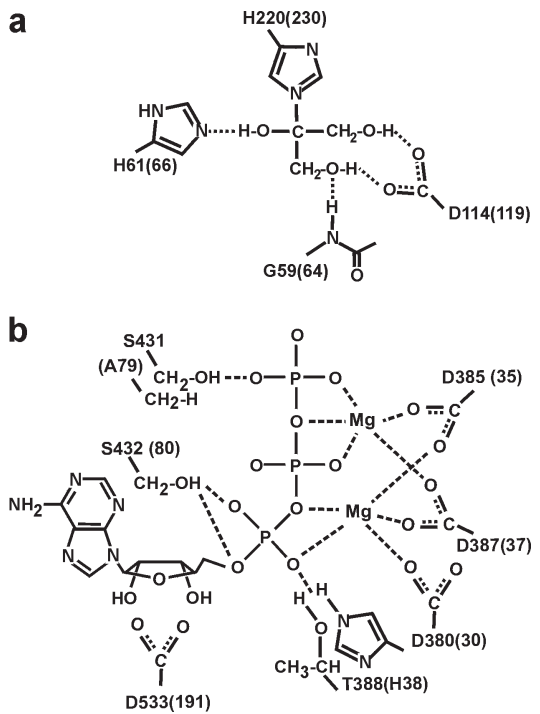

Figure 4. Ligand-protein interactions. (a) Structure of the hemiaminal between Dha and $\mathrm{N} \varepsilon 2$ of histidine, and hydrogen bonds to invariant His and Asp. (b) Hydrogen- and metal-binding interactions between ATP and conserved residues of the nucleotide binding site [62]. Numbers refer to residues of the C. freundii kinase, numbers in parenthesis to the E. coli kinase. H38 is invariant in the DhaL subunits of PEP-dependent kinases, T388 is conserved in most ATP-dependent kinases. 
and protein kinases $[69,70]$. First, the four loops are centred between $\alpha$-helices and not in $\beta$-hairpins or $\beta$ - $\alpha$ loops. And second, the loops do not contain aromatic residue or carboxy terminal lysines, which in the other ATP-binding proteins are in contact with the nucleotide.

The binding sites for Dha and ATP are $14 \AA$ apart in the crystal structure (Fig. 3a) and it is not clear how they reorient for in-line phosphoryltransfer. One way of bringing ATP and Dha together is that the two domains are freely mobile in solution [62]. Another possibility is tilting of the nucleotide-binding site by a shear deformation of the helix bundle along the barrel axis combined with the elliptical compression of the barrel in the direction of the K-domain.

The DhaK subunit of the PEP-dependent E. coli Dha kinase assumes the same fold as the $C$. freundii counterpart [63]. The only obvious difference is a $\beta$-ribbon of 25 residues inserted in the carboxy terminal subdomain of $E$. coli DhaK. The $\beta$-ribbon folds back to cap the edge of the $\beta$-sheet in the amino terminal $\alpha / \beta$ fold (Fig. 3b). It has been proposed that $\beta$-ribbons prevent the association between hydrophobic edge strands and concomitant protein aggregation $[71,72]$. The $\beta$-ribbon is present only in a small subgroup of PEP-dependent Dha kinases, which utilize large multidomain DhaM subunits as phosphorylgroup donors (see below).

The E. coli DhaL subunit is expected to assume the same fold as the L-domain of DAK (Fig. 3a). DhaL also binds a nucleotide, which, however, is retained during all purification steps. The exchange half-life of ADP in complex with DhaL is 100 min, five times the generation-time of a fast growing E. coli! And the removal of the nucleotide by sequestration of $\mathrm{Mg}^{2+}$ with EDTA reduces the thermal unfolding temperature of DhaL from 65 to $40{ }^{\circ} \mathrm{C}$ [61]. ADP is not exchanged for ATP in E. coli DhaL - unlike in any other kinase with known function - but is rephosphorylated in situ by the DhaM subunit. The nucleotide no longer is a substrate of DhaL but a coenzyme with the function of a phosphoryl group binding amino acid. This conversion of a substrate-binding site into a cofactor-binding site is a remarkable example of functional evolution [61].

DhaM is the third subunit of PEP-dependent Dha kinases [53]. In most species DhaM consists of a single domain that is homologous to the amino terminal domain of the IIAB $^{\text {Man }}$ subunit of the E. coli mannose transporter [64, $73,74]$. Occasionally and as shown below, DhaM contains additional domains similar to the phosphotransferase subunits HPr and enzyme I of the PTS $[64,73,74]$.

\section{Control of $d h a$ operon transcription by Dha kinases}

DhaK and DhaL homologs also occur in operons for putative transcription factors. These operons are adjacent and often divergently oriented to bona fide dha operons suggesting that they control Dha kinase expression. The molecular mechanisms by which Dha kinase subunits function as coactivators of transcription has been elucidated in E. coli (Fig. 5). Genetic and biochemical studies showed that the dha operon is controlled by DhaR and the two kinase subunits DhaK and DhaL $[60,75]$. DhaR belongs to the family of enhancer-binding proteins (EBPs) that activate the sigma RNA polymerase complex by direct contact and by causing DNA looping [76-78]. EBPs usually consist of three domains, a DNA-binding, a $\mathrm{AAA}^{+}$ATPase and a receiver domain for low-molecular and protein ligands. E. coli DhaR stimulates the transcription of the $d h a$ operon from a $\sigma 70$ promoter and autorepresses transcription of the dhaR gene. DhaK and DhaL antagonistically act as corepressor and coactivator of DhaR (Fig. 5). In the presence of Dha, when the phosphoryl group is transferred from DhaL::ATP to Dha, the now dephosphorylated DhaL::ADP binds to the DhaR receiver domain and activates the expression of the dha operon. In the absence of Dha, DhaL::ADP is rephosphorylated by DhaM to DhaL::ATP, which does not bind to DhaR. If DhaM or EI are inactivated by a mutation DhaL::ADP remains in the dephosphorylated state and the dha operon is expressed constitutively $[60,75]$. DhaK competes with DhaL for binding to DhaR and thus tunes down the effect of DhaL::ADP. Therefore transcription of the dha operon overshoots when DhaK and DhaM or DhaK and EI are inactivated together $[60,75]$. The novelty of this mechanism is (i) the formation of a complex between a transcription factor and two enzyme subunits and (ii) the coupling of transcription activation with turnover and not only binding of the substrate. The dou-

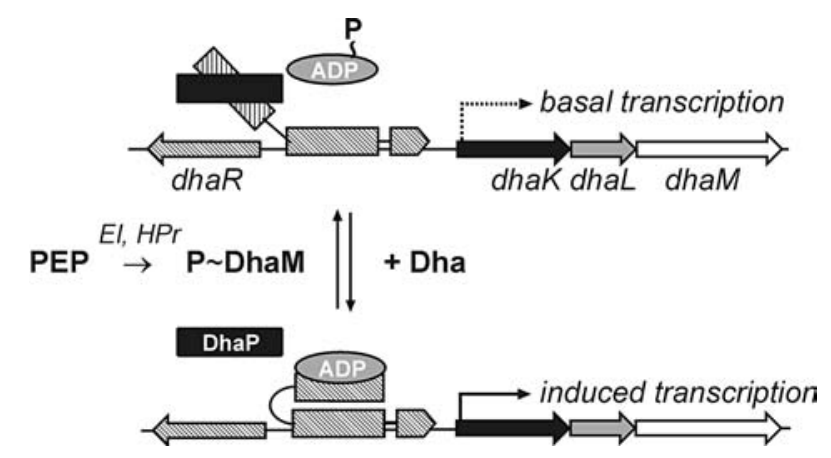

Figure 5. Model of Dha kinase mediated transcription control. E. coli DhaR (diagonally striped), the activator of the dhaKLM operon, consists of a receiver domain, an AAA+-ATPase domain and a DNA-binding helix-turn-helix domain. DhaL::ADP (grey) is the coactivator of DhaR, DhaK (black) an antagonist of DhaL::ADP. By binding to DhaK, the inducer Dha reduces the affinity of DhaK for DhaR. By dephosphorylation of DhaL::ATP, Dha increases the affinity of DhaL::ADP for DhaR. The $\sigma 70$ initiation factor that directs the RNA polymerase to the promoter site and the RNA polymerase are not shown. The dhaR gene is divergently transcribed from a constitutively active promoter which in E. coli is repressed by DhaR [75]. 
ble-check for binding and turnover may increase the selectivity and the sensitivity of control [75]. It is not known whether the DhaL-DhaR complex acts directly by allosteric activation of the $\sigma$ initiation factor and the RNA polymerase or indirectly by DNA bending.

In Lactococcus lactis an operon for a DhaK homolog (Swiss-Prot entry Q9CIW0), and a protein (Q9CIV9) with the predicted fold of a tetracyclin repressor [79] is found next to the bona fide dha operon. In Sinorhizobium meliloti an operon for a homolog of DhaL (Q92WN5) and a twodomain protein, Q92WN4, follow the genes for bona fide DhaK (Q92WN2) and DhaL (Q92WN3) subunits. The amino terminal domain of Q92WN4 has 27\% sequence identity with SorC (P37078), the regulator of the L-sorbose operon of K. pneumonia, and with DeoR (P39140), the regulator of the deoxyribonucleoside and deoxyribose utilization operon dra-nupC-pdp of Bacillus subtilis. The carboxy terminal DhaK-like domain of Q92WN4 has 41\% sequence identity with the S. meliloti DhaK (Q92WN2) subunit, and the DhaL paralogs (Q92WN5 and Q92WN3) share $33 \%$ sequence identity. It is not known how these transcription regulators function or what the role of the associated kinase subunits may be.

\section{Distribution and phylogeny of Dha kinases}

A protein-protein BLAST (blastp at NCBI) with DhaK (DhaL) of E. coli as the input generated 169 (161) hits from 115 (117) bacterial species and 70 (56) hits from 39 (34) eukaryotes. With the single-domain DhaM (Q9CIV6) of L. lactis as input, the output contained 87 hits from 78 bacterial species and none from eukaryotes. 60 hits from bacterial and eukaryotic species were selected for closer inspection and classified into three families (1-3) according to the organization of the operon, the subunit composition of the gene product (Fig. 6), the phylogenetic relationship (Fig. 7) and the predicted function: (i) Dha kinases, (ii) DhaK and DhaL homologs (paralogs) associated with putative transcription regulators and (iii) proteins of unknown function with circularly permuted domain order.

The kinase family (i) can be further divided into four groups (a-d, Figs. 6 and 7): (a) Single-subunit ATP-dependent Dha kinases like the $C$. freundii kinase described above. This group also includes all Dha kinases of yeasts, plants and animals (EC 2.7.1.28) and the triokinases (EC 2.7.1.29), which were defined based on their specificity for glyceraldehyde but probably are not an enzyme class of their own. The $C$. freundii kinase displays more sequence identity $(40 \%)$ with human than with the yeast (Pichia pastoris) kinases (30\%). Altogether the yeast kinases form a large, separate branch within group A, which for lack of space has been omitted from Figure 7. (b) PEP-dependent Dha kinases that consist of DhaK,

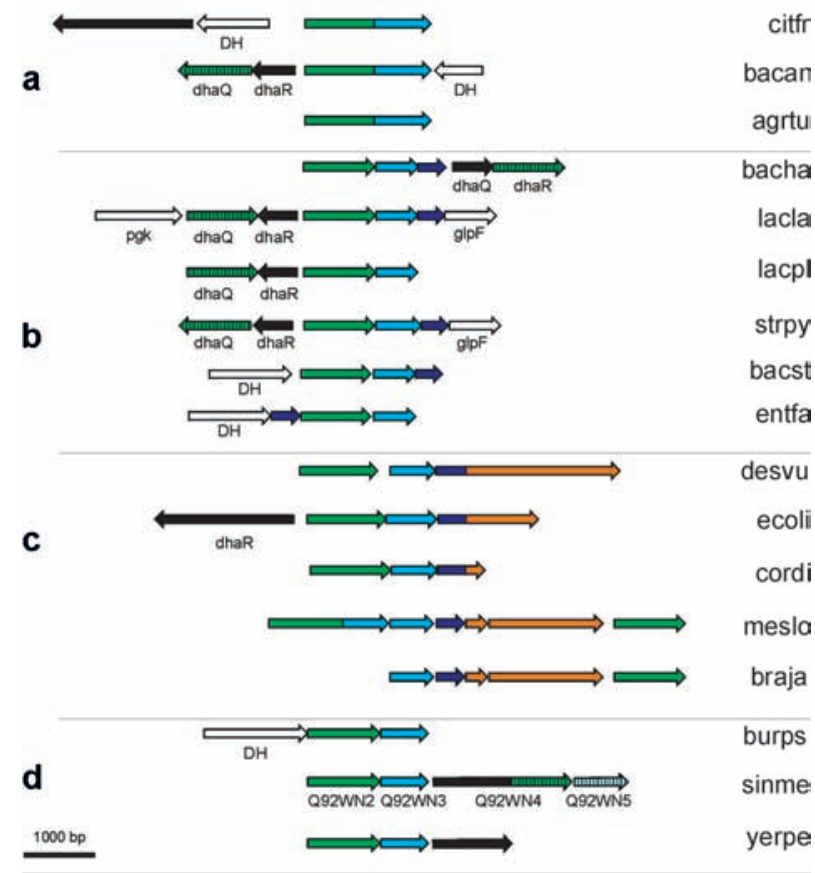

Figure 6. Structures of dha operons. (a) Genes for ATP-dependent Dha kinases with and without divergently transcribed genes for DhaR. (b) Genes for PEP-dependent kinases with and without genes for Tet-R-like (dhaR) and DhaK-like (dhaQ) transcription regulators. (c) PEP-dependent Dha kinases with large multidomain DhaM subunits and genes for EI and HPr of the PTS. The M. loti locus contains genes for PTS- and ATP-dependent kinases. (d) Operons for Dha kinases without a DhaM subunit and associated transcription regulators of the SorC/DeoR-type. The domains/subunits and their colours are: DhaK (green), DhaL (cyan), DhaM IIA-domain (blue), PTS domains (orange), DNA-binding proteins (black), DhaK-like (dhaQ; green striped). DH refers to dehydrogenase, glpF to glycerol facilitator. The labels $a-d$ refer to the clusters in the comparative tree (Fig. 7). For abbreviations, see Fig. 7.

DhaL and a 'small' single-domain DhaM subunits. They form the largest group of bacterial Dha kinases. (c) PEPdependent Dha kinases that contain 'large' multidomain DhaMs. Their DhaMs contain in addition to the obligatory IIA ${ }^{\text {Man }}$-like domain additional domains similar to HPr and enzyme I (EI) of the PTS (Fig. 6). Two-domain (IIA-HPr) DhaMs occur in some Actinobacteria [Corynebacterium diphtheriae (Q6NED9)], three-domain (IIAHPr-EI(N) DhaMs in E. coli, and four-domain [IIA-HPr$\mathrm{EI}(\mathrm{N})-\mathrm{EI}(\mathrm{C})]$ DhaMs with full-length (PEP-binding) EI in Desulvofibrio vulgaris (Q72DE8) and some other strains of the $\delta$ and $\gamma$ subdivision of proteobacteria. It is noteworthy that all the $\beta$-hairpin-containing DhaKs (Fig. 3b) are associated with such three- and four-domain DhaMs. (d) The fourth group contains two types of Dha kinases: (i) Two-subunit kinases (DhaK and DhaL) encoded by discistronic operons without a gene for a DhaM subunit. Their DhaLs have an invariant histidine in loop 1 of the nucleotide-binding site (LGDGDHG, Fig. 4b) like the PEP-dependent kinases of groups B and C. (ii) Kinases that by their domain architecture resemble ATP-depen- 


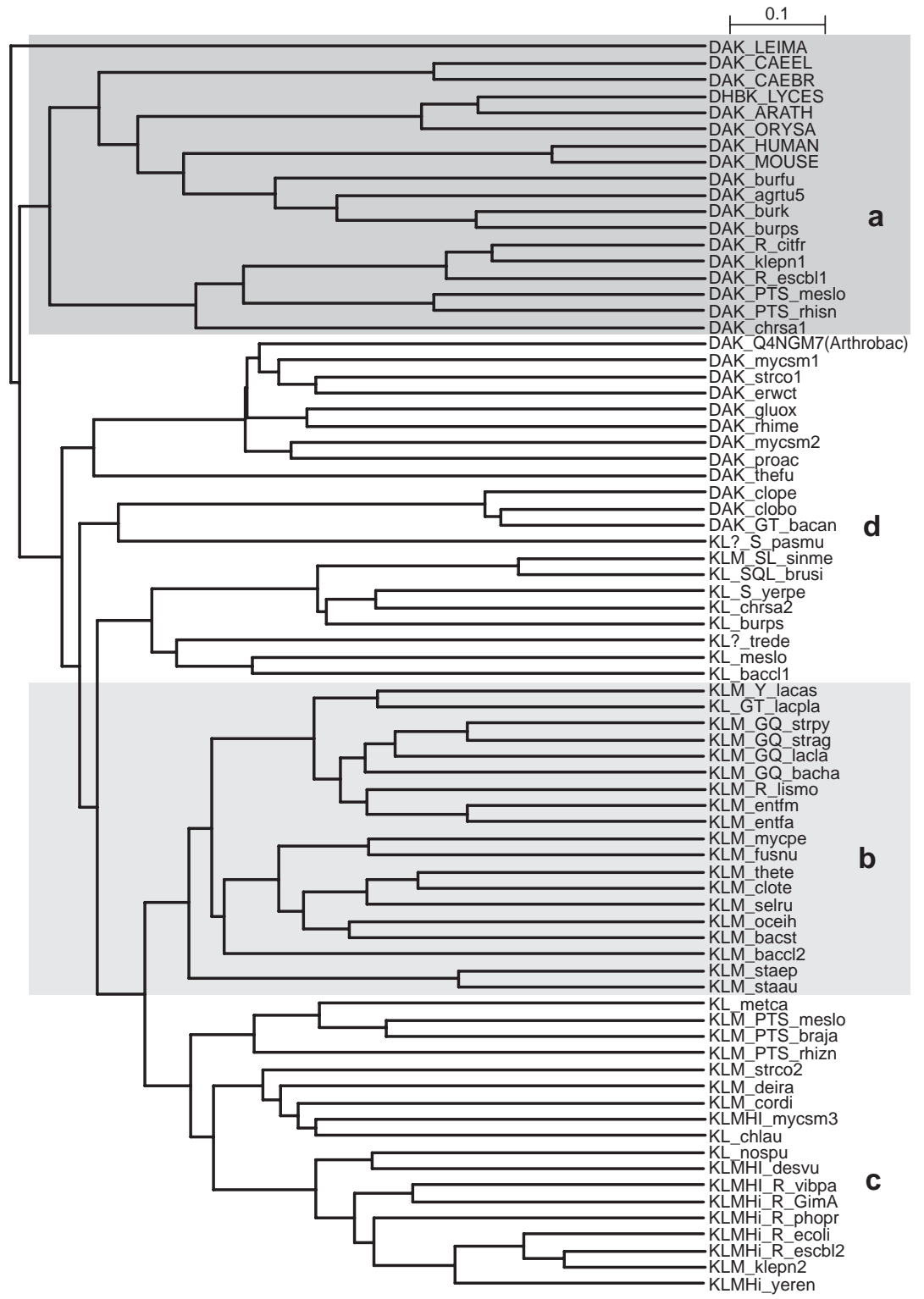

Figure 7. Phylogenetic tree of Dha kinases. DhaK and DhaL subunits encoded in an operon were treated as a unit. DAK refers to two-domain Dha kinases, DHBK to 3,4-dihydroxy-2-butanone kinase [51], KL to two-subunit Dha kinases without a DhaM subunit and KLM to three subunit kinases. M indicates single-domain (IIA) DhaM subunits, MH two-domain (IIA-HPr), MHi three-domain [IIA-HPr-EI(N)] and MHI four-domain [IIA-HPr-EI(N)-EI(C)] DhaM subunits. PTS indicates that genes for PTS proteins (EI and HPr) are associated with the dha operon as shown in Figure 6. G (DhaR,TetR-like), Q (DhaK-like), R (AAA+), S (SorC-like) and L (DhaL-like) indicate that transcription factors are associated with the dha operon as shown in Figure 6. X,Y and ? refer to genes of unknown function. Numbers after the species abbreviation indicate that their genomes encode more than one Dha kinase. Bar, 0.1 replacement per position. The sequences were aligned with the program MULTALIGN (www.toulouse.inra.fr/multalin.html) [93] and clustered with TREETOP (www.genebee.msu.su/services/phtree_full.html) [94] with alignment parameters set to default values. The species abbreviations are: Leishmania (LEIMA); Caenorhabiditis (CAE); Arabidopsis (ARATH); tomato (LYCES); rice (ORYSA); Agrobacterium tumfaciens (agrtu); Bacillus anthracis (bacan); Bacillus halodurans (bacha); Bacillus clausii (baccl); Bacillus stearothermophilus (bacst); Bradyrhizobium japonicum (braja); Brucella suis (brusi); Burkholderia pseudomallei (burps); Chloroflexus aurantiacus (chlau); Chromohalobacter salexigens (chrsa); Citrobacter freundii (citfr); Clostridium perfringens (clope); Clostridium botulinum (clobo); Clostridium tetani (clote); Corynebacterium diphtheriae (cordi); Deinococcus radiodurans (deira); Desulfovibrio vulgaris (desvu); Erwinia carotovora (erwct); Escherichia coli K1 genetic island (GimA); Escherichia coli K12 (ecoli); Escherichia blattae (escbl); Enterococcus faecalis (entfa); Enterococcus faecium (entfm); Fusobacterium nucleatum (fusnu); Gluconobacter oxidans (gluox); Klebsiella pneumoniae (klepn); Lactococcus lactis (lacla); Lactococcus plantarum (lacpl); Listeria monocytogenes (lismo); Pasteurella multocida (pasmu); Photobacterium profundum (phopr); Mesorhizobium loti (meslo); Methylococcus capsulatus (metca); Mycoplasma penetrans (mycpe); Mycobacterium smegmatis (mycsm); Nostoc punctiforme (nospu); Oceanobacillus iheyensis (oceih); Rhizobium meliloti (rhime); Rhizobium sp. (rhizn); Selonomonas ruminantium (selru); Sinorhizobium meliloti (sinme); Staphylococcus aureus (staau); Staphylococcus epidermidis (staep); Streptomyces coelicolor (strco); Streptococcus agalactiae (strag); Streptococcus pyogenes (strpy); Thermoanaerobacter tengcongensis (thete); Treponema denticola (trede); Vibrio parahaemolyticus (vibpa); Yersinia enterocolitica (yeren); Yersinia pestis (yerpe). 
dent kinases, but which by their sequence are more similar to the PEP-dependent forms. The bacterial strains encoding such 'incomplete' Dha kinases often also contain only a reduced PTS with genes for homologs of EI, HPr and IIA proteins [80] but not for carbohydrate transporters (enzymes II). The function of these incomplete kinases and whether other IIA-like proteins can complement the missing DhaMs is not known.

The second family of DhaK and DhaL paralogs associated with transcription regulators (DhaRs) of the TetR and DeoR families has been described above. They form a small branch of their own within the $\mathrm{D}$ group (not shown in Figure 7). The 'regulatory' orthologs (termed DhaQ) from different strains are more closely related to each other (50-65\% sequence identity) than to the catalytic DhaK paralogs (identity $40-45 \%$ ). It thus appears that the duplication and the functional diversification of the ancestral dhaK gene preceded the evolutionary separation of the modern carriers of these paralogous genes.

The third family comprises the DegV-like proteins (Pfam accession number 02645), whose function is not known. They have a circularly permuted domain order. The fold of the amino terminal domain is similar to DhaL. The fold of the carboxy terminal domain is similar to the fatty acid binding protein TM841 of Thermotoga maritima (PDB code 1MGP [81]), and TM841 in turn shares the core fold with DhaK [62,63], as shown in the topological diagrams of Figure 3c. The difference between DhaK and TM841 lies in two extra secondary structure elements and in the chemistry of their ligand binding sites. TM841 and - by prediction - the carboxy terminal domains of DegV proteins have an extra $\alpha / \beta$ fold inserted between $\beta 2$ and $\alpha 2$ of the core, while DhaK has two extra $\beta$-strands and one $\alpha$-helix (a dimerization helix) appended to the amino terminus. The ligands, Dha in DhaK and a fatty acid in TM841, occupy the same binding pocket and are both hydrogen bonded to the first topological switch point of the amino terminal $\alpha / \beta$ subdomain. The residues, involved in ligand binding are, however, different. It is not known whether the DhaL-like domain of DegV proteins has kinase activity or what the substrate and molecular function of the DhaK-like domain might be.

\section{Biological function of Dha kinases and open questions}

While the structure and molecular function of the PEPdependent $E$. coli Dha kinase and of the ATP-dependent C. freundii kinase are reasonably well understood, much less is known about the physiological role of Dha kinases in living cells. Two well-characterized functions, namely (i) catabolism of glycerol and (ii) methanol assimilation (methylotrophy), have been described above (Fig. 1). Two additional functions (iii) detoxification (chemical stress response) and (iv) control of gene expression will be discussed in this section. Glyceraldehyde and Dha can crosslink proteins and by autooxidation produce superoxide radicals [82, 83], and Dha has been shown to enhance the mutation rate in SOD-deficient E. coli [84-86]. Molin et al. [87] demonstrated that Dha is toxic to Saccharomyces cerevisiae and that detoxification is dependent on a functional Dha kinase. Deletion of the two genes for Dak1p and Dak2p made cells hypersensitive, while overexpression made them more tolerant to Dha. Dha toxicity was dependent on cosubstrates and salt conditions, which led to the proposition that detoxification of Dha might be a part of the physiological response to Dha-dependent stress conditions. It is therefore reasonable to assume that Dha kinases clean up the short-chain carbonyl compounds that may be formed as side products of the carbohydrate metabolism (aldol cleavage of ketohexoses and ketopentoses or oxidation of glycerol). The rate of Dha formation by non-specific oxidation may be increased in organisms that under osmotic stress accumulate glycerol in high concentrations $[88,89]$. Owing to hemiaminal formation, Dha kinases can remove traces of Dha even in the presence of an excess of glycerol [66], which glycerol kinases, for comparison, cannot.

The substrate spectrum of some 'Dha kinases' may be broader than suggested by most of the published reports. A recent and unexpected finding is that FMN-cyclase, an enzyme that catalyses the formation of cyclic FMN (riboflavin $4^{\prime}, 5^{\prime}$-cyclic phosphate) from FAD, is identical with the human Dha kinase (SWISS-Prot Q3LXA3, Q9BVA7) [90]. Cyclase and kinase activity were copurified from rat liver extracts as well as with the human recombinant protein. Kinase activity was six times stronger than cyclase activity, but FAD, ATP and Dha were mutually competitive inhibitors [90]. While the ADP moiety of FAD in principle can be accommodated in the nucleotide binding site, it is not clear at all how the riboflavin moiety can bind to the L- or K-domain.

The physiological role of Dha kinases in transcription control is still far from understood. One answer may be found in bacteria that contain both a PEP-dependent and an ATP-dependent kinase, for instance Escherichia blattae [91], K. pneumoniae, Streptomyces coelicolor and probably also $C$. freundii. DhaR of E. coli controls not only the expression of the $E$. coli but also of the $C$. freundii kinase [75], suggesting that the PEP-dependent form functions as 'sensor kinase' for the control of the ATP-dependent 'metabolic kinase'. A further hint that this sensor kinase may also control other biological processes is provided by the $20-\mathrm{kb}$ genetic island of meningitic $E$. coli $K 1$ (GimA) [92]. This island contains among others the ibeA virulence gene (invasin) that contributes to the pathogenesis of $E$. coli meningitis, three genes for a PEP-dependent Dha kinase ( $p t n C, p t n K, p t n P$ ) and the gene ibeR for a homolog of the E. coli DhaR. Of all the potential promoter/ 
operator sequences on GimA, the putative promoters of ibeA (Q9FCY5) and ptnC (Q8VP37) display the strongest similarity to the promoter/operator region of the $E$. coli dha operon [75]. It is therefore possible that IbeR controls the $i b e A$ virulence gene and that the PEP-dependent Dha kinase serves as the sensor for an environmental stimulus at the blood-brain barrier. However, this hypothesis has not yet been tested.

Acknowledgements. Cited works carried out by the authors of this review were supported by grant 3100-063420 from the Swiss National Science Foundation, the Ciba-Geigy Jubiläumsstiftung and the Roche Foundation.

1 Hirabayashi J. (1996) On the origin of elementary hexoses. Q. Rev. Biol 71: 365-380

2 Enders D., Voith M. and Lenzen A. (2005) The dihydroxyacetone unit-a versatile $\mathrm{C}(3)$ building block in organic synthesis. Angew. Chem. Int. Ed Engl. 44: 1304-1325

3 Slepokura K. and Lis T. (2004) Crystal structures of dihydroxyacetone and its derivatives. Carbohydr. Res. 339: 1995-2007

4 Nguyen B. C. and Kochevar I. E. (2003) Influence of hydration on dihydroxyacetone-induced pigmentation of stratum corneum. J. Invest. Dermatol. 120: 655-661

5 Tessier F. J., Monnier V. M., Sayre L. A. and Kornfield J. A. (2002) Triosidines: novel maillard reaction products and crosslinks from the reaction of triose sugars with lysine and arginine residues. Biochem. J. 369: 705-719

6 Fu J. M., Dusza S. W. and Halpern A. C. (2004) Sunless tanning. J Am. Acad Dermatol. 50: 706-713

7 Faurschou A., Janjua N. R. and Wulf H. C. (2004) Sun protection effect of dihydroxyacetone. Arch. Dermatol. 140: 886-887

8 Hunter I. R., Walden M. K., Scherer J. R. and Lundin R. E. (1969) Preparation and properties of 1,4,5,6-tetrahydro-2 acetopyridine. A cracker-odor constituent of bread aroma. Cereal Chemistry 46: 189-195

9 Adams A., Tehrani K. A., Kersiene M. and De Kimpe N. (2004) Detailed investigation of the production of the bread flavor component 6-acetyl-1,2,3,4-tetrahydropyridine in proline/1,3dihydroxyacetone model systems. J. Agric. Food Chem. 52: 5685-5693

10 Daniel R., Stuertz K. and Gottschalk G. (1995) Biochemical and molecular characterization of the oxidative branch of glycerol utilization by Citrobacter freundii. J. Bacteriol. 177: 43924401

11 Forage R. G. and Lin E. C. (1982) DHA system mediating aerobic and anaerobic dissimilation of glycerol in Klebsiella pneumoniae NCIB 418. J. Bacteriol. 151: 591-599

12 Gancedo C., Llobell A., Ribas J. C. and Luchi F. (1986) Isolation and characterization of mutants from Schizosaccharomyces pombe defective in glycerol catabolism. Eur. J. Biochem. 159: $171-174$

13 Johnson E. A., Burke S. K., Forage R. G. and Lin E. C. (1984) Purification and properties of dihydroxyacetone kinase from Klebsiella pneumoniae. J. Bacteriol. 160: 55-60

14 Deppenmeier U., Hoffmeister M. and Prust C. (2002) Biochemistry and biotechnological applications of Gluconobacter strains. Appl. Microbiol. Biotechnol. 60: 233-242

15 Bauer R., Katsikis N., Varga S. and Hekmat D. (2005) Study of the inhibitory effect of the product dihydroxyacetone on Gluconobacter oxydans in a semi-continuous two-stage repeatedfed-batch process. Bioprocess. Biosyst. Eng. 1-7

16 Kimura T., Takahashi M., Yoshihara K., Furuichi T., Suzuki K., Imai K. et al. (1998) Cloning and characterization of two genes encoding dihydroxyacetone kinase from Schizosaccharomyces pombe IFO 0354. Biochim. Biophys. Acta 1442: 361-368
17 Luers G. H., Advani R., Wenzel T. and Subramani S. (1998) The Pichia pastoris dihydroxyacetone kinase is a PTS1-containing, but cytosolic, protein that is essential for growth on methanol. Yeast 14: 759-771

18 Stewart M. Q., Esposito R. D., Gowani J. and Goodman J. M. (2001) Alcohol oxidase and dihydroxyacetone synthase, the abundant peroxisomal proteins of methylotrophic yeasts, assemble in different cellular compartments. J. Cell Sci. 114: 2863-2868

19 Waites M. J. and Quayle J. R. (1981) The interrelation transketolase and dihydroxyacetone synthase activities in the methylotrophic yeast Candida boidinii. J. Gen. Microbiol. 124: 309316

20 de Koning W. and Harder W. (1992) Methanol-utilizing yeasts. In: Methane and Methanol Utilizers, pp. 207-244, Murrell J. C. and Dalton H. (eds.), Plenum Press, New York

21 Dijkhuizen L., Levering P. R. and de Vries G. E. (1992) The physiology and biochemistry of aerobic methanol-utilizing Gram-negative and Gram-positive bacteria. In: Methane and Methanol Utilizers, pp. 149-181, Murrell J. C. and Dalton H. (eds.), Plenum Press, New York

22 Hanson R. S. and Hanson T. E. (1996) Methanotrophic bacteria. Microbiol. Mol. Biol. Rev. 60: 439-471

23 Park S. W., Hwang E. H., Park H., Kim J. A., Heo J., Lee K. H. et al. (2003) Growth of mycobacteria on carbon monoxide and methanol. J. Bacteriol. 185: 142-147

24 Ro Y. T., Eom C. Y., Song T., Cho J. W. and Kim Y. M. (1997) Dihydroxyacetone synthase from a methanol-utilizing carboxydobacterium, Acinetobacter sp. strain JC1 DSM 3803. J. Bacteriol. 179: 6041-6047

25 Johnson A. E. and Tanner M. E. (1998) Epimerization via carbon-carbon bond cleavage. L-ribulose-5-phosphate 4-epimerase as a masked class II aldolase. Biochemistry 37: 57465754

26 Lee L. V., Poyner R. R., Vu M. V. and Cleland W. W. (2000) Role of metal ions in the reaction catalyzed by L-ribulose-5-phosphate 4-epimerase. Biochemistry 39: 4821-4830

27 Jeffery J. and Jornvall H. (1983) Enzyme relationships in a sorbitol pathway that bypasses glycolysis and pentose phosphates in glucose metabolism. Proc. Natl. Acad. Sci. USA 80: 901-905

28 Antinozzi P. A., Ishihara H., Newgard C. B. and Wollheim C. B. (2002) Mitochondrial metabolism sets the maximal limit of fuel-stimulated insulin secretion in a model pancreatic beta cell: a survey of four fuel secretagogues. J. Biol. Chem. 277: $11746-11755$

29 Azzout B., Chanez M., Bois-Joyeux B. and Peret J. (1984) Gluconeogenesis from dihydroxyacetone in rat hepatocytes during the shift from a low protein, high carbohydrate to a high protein, carbohydrate-free diet. J. Nutr. 114: 2167-2178

30 Cortez M. Y., Torgan C. E., Brozinick J. T., Miller R. H. and Ivy J. L. (1991) Effects of pyruvate and dihydroxyacetone consumption on the growth and metabolic state of obese Zucker rats. Am. J. Clin. Nutr. 53: 847-853

31 Jenkins B. T. and Hajra A. K. (1976) Glycerol kinase and dihydroxyacetone kinase in rat brain. J. Neurochem. 26: 377-385

32 Taguchi T., Murase S. and Miwa I. (2002) Glyceraldehyde metabolism in human erythrocytes in comparison with that of glucose and dihydroxyacetone. Cell Biochem. Funct. 20: 223-226

33 Ivy J. L. (1998) Effect of pyruvate and dihydroxyacetone on metabolism and aerobic endurance capacity. Med. Sci Sports Exerc. 30: 837-843

34 Stanko R. T. and Arch J. E. (1996) Inhibition of regain in body weight and fat with addition of 3-carbon compounds to the diet with hyperenergetic refeeding after weight reduction. Int. J. Obes. Relat. Metab. Disord. 20: 925-930

35 Hurley J. H., Faber H. R., Worthylake D., Meadow N. D., Roseman S., Pettigrew D. W. et al. (1993) Structure of the regulatory complex of Escherichia coli III(Glc) with glycerol kinase. Science 259: 673-677 
36 Yeh J. I., Charrier V., Paulo J., Hou L., Darbon E., Claiborne A. et al. (2004) Structures of enterococcal glycerol kinase in the absence and presence of glycerol: correlation of conformation to substrate binding and a mechanism of activation by phosphorylation. Biochemistry 43: 362-373

37 Deutscher J. and Sauerwald H. (1986) Stimulation of dihydroxyacetone and glycerol kinase activity in Streptococcus faecalis by phosphoenolpyruvate-dependent phosphorylation catalyzed by enzyme I and HPr of the phosphotransferase system. J. Bacteriol. 166: 829-836

38 Hayashi S. I. and Lin E. C. (1967) Purification and properties of glycerol kinase from Escherichia coli. J. Biol. Chem. 242: $1030-1035$

39 Webb M. R. (1992) A continuous spectrophotometric assay for inorganic phosphate and for measuring phosphate release kinetics in biological systems. Proc. Natl. Acad. Sci. USA 89: 4884-4887

40 Weinhouse H. and Benziman M. (1976) Phosphorylation of glycerol and dihydroxyacetone in Acetobacter xylinum and its possible regulatory role. J. Bacteriol. 127: 747-754

41 Ruch F. E., Lengeler J. and Lin E. C. (1974) Regulation of glycerol catabolism in Klebsiella aerogenes. J. Bacteriol. 119: $50-56$

42 Bystrykh L. V., de K. W. and Harder W. (1990) Triokinase from Candida boidinii KD1. Methods Enzymol. 188: 445-451

43 Hofmann K. H. and Babel W. (1981) Dihydroxyacetone kinase of methanol-assimilating yeasts. II. Partial purification and some properties of dihydroxyacetone kinase from Candida methylica. Z. Allg. Mikrobiol. 21: 219-224

44 Hofmann K. H. and Babel W. (1990) Glycerone kinase from Candida methylica. Methods Enzymol. 188: 451-455

45 Itoh N., Tujibata Y. and Liu J. Q. (1999) Cloning and overexpression in Escherichia coli of the gene encoding dihydroxyacetone kinase isoenzyme I from Schizosaccharomyces pombe, and its application to dihydroxyacetone phosphate production. Appl. Microbiol. Biotechnol. 51: 193-200

46 Molin M., Larsson T., Karlsson K. A. and Blomberg A. (2003) Fragmentation of dihydroxyacetone kinase 1 from Saccharomyces cerevisiae indicates a two-domain structure. Proteomics 3: 752-763

47 Wang Z. X., Kayingo G., Blomberg A. and Prior B. A. (2002) Cloning, sequencing and characterization of a gene encoding dihydroxyacetone kinase from Zygosaccharomyces rouxii NRRL2547. Yeast 19: 1447-1458

48 van der Klei I. J., van der Heide M., Baerends R. J., Rechinger K. B., Nicolay K., Kiel J. A. et al. (1998) The Hansenula polymorpha per6 mutant is affected in two adjacent genes which encode dihydroxyacetone kinase and a novel protein, Pak1p, involved in peroxisome integrity. Curr. Genet. 34: 1-11

49 Huang J. M., Wei Y. F., Kim Y. H., Osterberg L. and Matthews H. R. (1991) Purification of a protein histidine kinase from the yeast Saccharomyces cerevisiae. The first member of this class of protein kinases. J. Biol. Chem. 266: 9023-9031

50 Lerner H. R., Sussman I. and Avron M. (1980) Characterization and partial purification of dihydroxyacetone kinase in $\mathrm{Du}$ naliella salina. Biochim. Biophys. Acta 615: 1-9

51 Herz S., Kis K., Bacher A. and Rohdich F. (2002) A tomato enzyme catalyzing the phosphorylation of 3,4-dihydroxy-2-butanone. Phytochemistry 60: 3-11

52 Miwa I., Kito Y. and Okuda J. (1994) Purification and characterization of triokinase from porcine kidney. Prep. Biochem. 24: 203-223

53 Gutknecht R., Beutler R., Garcia Alles L. F., Baumann U. and Erni B. (2001) The dihydroxyacetone kinase of Escherichia coli utilizes a phosphoprotein instead of ATP as phosphoryldonor. EMBO J. 20: 2480-2486

54 Jin R. Z., Tang J. C. and Lin E. C. (1983) Experimental evolution of a novel pathway for glycerol dissimilation in Escherichia coli. J. Mol. Evol. 19: 429-436
55 Postma P. W., Lengeler J. W. and Jacobson G. R. (1996) Phosphoenolpyruvate: carbohydrate phosphotransferase systems. In: Escherichia coli and Salmonella: Cellular and Molecular Biology, pp. 1149-1174, Neidhardt F. C., Curtiss R., Ingraham J. L., Lin E. C. C., Low K. B., Magasanik B. (eds.), ASM Press, Washington, DC

56 Saier M. H., Hvorup R. N. and Barabote R. D. (2005) Evolution of the bacterial phosphotransferase system: from carriers and enzymes to group translocators. Biochem. Soc. Trans. 33: 220-224

57 Siebold C., Flükiger K., Beutler R. and Erni B. (2001) Carbohydrate transporters of the bacterial phosphoenolpyruvate: sugar phosphotransferase system (PTS). FEBS Lett. 504: 104-111

58 Jin R. Z. and Lin E. C.C. (1984) An inducible phosphoenolpyruvate: dihydroxyacetone phosphotransferase system in Escherichia coli. J. Gen. Microbiol. 130: 83-88

59 Paulsen I. T., Reizer J., Jin R. Z., Lin E. C. and Saier M. H. (2000) Functional genomic studies of dihydroxyacetone utilization in Escherichia coli. Microbiology 146: 2343-2344

60 Beutler R., Kämpfer U., Schaller J. and Erni B. (2001) Heterodimeric dihydroxyacetone kinase from a ptsI mutant of Escherichia coli. Microbiology 147: 249-250

61 Bachler C., Flukiger-Bruhwiler K., Schneider P., Bahler P. and Erni B. (2005) From ATP as substrate to ADP as coenzyme: functional evolution of the nucleotide binding subunit of dihydroxyacetone kinases. J. Biol. Chem. 280: 18321-18325

62 Siebold C., Arnold I., Garcia-Alles L. F., Baumann U. and Erni B. (2003) Crystal structure of the Citrobacter freundii dihydroxyacetone kinase reveals an eight-stranded alpha-helical barrel ATP-binding domain. J. Biol. Chem. 278: 48236-48244

63 Siebold C., Garcia-Alles L. F., Erni B. and Baumann U. (2003) A novel mechanism of covalent substrate binding in the X-ray structure of the DhaK subunit of the Escherichia coli dihydroxyacetone kinase. Proc. Natl. Acad. Sci. USA 100: 81888192

64 Nunn R. S., Markovic-Housley Z., Génovésio-Taverne J. C., Flükiger K., Rizkallah P. J., Jansonius J. N. et al. (1996) Structure of the IIA domain of the mannose transporter from Escherichia coli at $1.7 \AA$ resolution. J. Mol. Biol. 259: 502-511

65 Kinch L. N., Cheek S. and Grishin N. V. (2005) EDD, a novel phosphotransferase domain common to mannose transporter EIIA, dihydroxyacetone kinase, and DegV. Protein Sci. 14: 360-367

66 Garcia-Alles L. F., Siebold C., Nyffeler T. L., Flukiger-Bruhwiler K., Schneider P., Burgi H. B. et al. (2004) Phosphoenolpyruvate- and ATP-dependent dihydroxyacetone kinases: covalent substrate-binding and kinetic mechanism. Biochemistry 43: 13037-13045

67 Cheek S., Ginalski K., Zhang H. and Grishin N. V. (2005) A comprehensive update of the sequence and structure classification of kinases. BMC. Struct. Biol. 5: 6

68 Vetter I. R. and Wittinghofer A. (1999) Nucleoside triphosphate-binding proteins: different scaffolds to achieve phosphoryl transfer. Q. Rev. Biophys. 32: 1-56

69 Bossemeyer D. (1994) The glycine-rich sequence of protein kinases: a multifunctional element. Trends Biochem. Sci. 19: 201-205

70 Saraste M., Sibbald P. R. and Wittinghofer A. (1990) The Ploop - a common motif in ATP- and GTP-binding proteins. Trends Biochem. Sci. 15: 430-434

71 Richardson J. S. and Richardson D. C. (2002) Natural betasheet proteins use negative design to avoid edge-to-edge aggregation. Proc. Natl. Acad. Sci. USA 99: 2754-2759

72 Wang W. and Hecht M. H. (2002) Rationally designed mutations convert de novo amyloid-like fibrils into monomeric betasheet proteins. Proc. Natl. Acad. Sci. USA 99: 2760-2765

73 Jia Z. C., Vandonselaar M., Hengstenberg W., Quail J. W. and Delbaere L. T.J. (1994) The 1 center dot 6 angstrom structure of histidine-containing phosphotransfer protein Hpr from Streptococcus faecalis. J. Mol. Biol. 236: 1341-1355 
74 Liao D. I., Silverton E., Seok Y. J., Lee B. R., Peterkofsky A. and Davies D. R. (1996) The first step in sugar transport: crystal structure of the amino terminal domain of enzyme I of the $E$ coli PEP: sugar phosphotransferase system and a model of the phosphotransfer complex with HPr. Structure 4: 861-872

75 Bachler C., Schneider P., Bahler P., Lustig A. and Erni B. (2005) Escherichia coli dihydroxyacetone kinase controls gene expression by binding to transcription factor DhaR. EMBO J. 24: 283-293

76 Buck M., Gallegos M. T., Studholme D. J., Guo Y. and Gralla J. D. (2000) The bacterial enhancer-dependent sigma(54) $(\operatorname{sigma}(\mathrm{N}))$ transcription factor. J. Bacteriol. 182: 4129-4136

77 Rappas M., Schumacher J., Beuron F., Niwa H., Bordes P., Wigneshweraraj S. et al. (2005) Structural insights into the activity of enhancer-binding proteins. Science 307: 1972-1975

78 Zhang X., Chaney M., Wigneshweraraj S. R., Schumacher J., Bordes P., Cannon W. et al. (2002) Mechanochemical ATPases and transcriptional activation. Mol. Microbiol. 45: 895-903

79 Ramos J. L., Martinez-Bueno M., Molina-Henares A. J., Teran W., Watanabe K., Zhang X. et al. (2005) The TetR family of transcriptional repressors. Microbiol. Mol. Biol. Rev. 69: 326 356

80 Powell B. S., Court D. L., Inada T., Nakamura Y., Michotey V., Cui X. et al. (1995) Novel proteins of the phosphotransferase system encoded within the rpoN operon of Escherichia coli. Enzyme IIA ${ }^{\mathrm{Ntr}}$ affects growth on organic nitrogen and the conditional lethality of an era $^{\text {ts }}$ mutant. J. Biol. Chem. 270: 48224839

81 Schulze-Gahmen U., Pelaschier J., Yokota H., Kim R. and Kim S. H. (2003) Crystal structure of a hypothetical protein, TM841 of Thermotoga maritima, reveals its function as a fatty acidbinding protein. Proteins 50: 526-530

82 Benov L. and Beema A. F. (2003) Superoxide-dependence of the short chain sugars-induced mutagenesis. Free Radic. Biol. Med. 34: 429-433

83 Tessier F. J., Monnier V. M., Sayre L. A. and Kornfield J. A. (2003) Triosidines: novel Maillard reaction products and crosslinks from the reaction of triose sugars with lysine and arginine residues. Biochem. J. 369: 705-719

84 Benov L., Beema A. F. and Sequeira F. (2003) Triosephosphates are toxic to superoxide dismutase-deficient Escherichia coli. Biochim. Biophys. Acta 1622: 128-132
85 Feron V. J., Til H. P., de Vrijer F., Woutersen R. A., Cassee F. R. and van Bladeren P. J. (1991) Aldehydes: occurrence, carcinogenic potential, mechanism of action and risk assessment. Mutat. Res. 259: 363-385

86 Petersen A. B., Wulf H. C., Gniadecki R. and Gajkowska B. (2004) Dihydroxyacetone, the active browning ingredient in sunless tanning lotions, induces DNA damage, cell-cycle block and apoptosis in cultured HaCaT keratinocytes. Mutat. Res. 560: $173-186$

87 Molin M., Norbeck J. and Blomberg A. (2003) Dihydroxyacetone kinases in Saccharomyces cerevisiae are involved in detoxification of dihydroxyacetone. J. Biol. Chem. 278: 1415-1423

88 Akhtar N., Blomberg A. and Adler L. (1997) Osmoregulation and protein expression in a pbs2delta mutant of Saccharomyces cerevisiae during adaptation to hypersaline stress. FEBS Lett. 403: $173-180$

89 Norbeck J. and Blomberg A. (1997) Metabolic and regulatory changes associated with growth of Saccharomyces cerevisiae in $1.4 \mathrm{M} \mathrm{NaCl}$. Evidence for osmotic induction of glycerol dissimilation via the dihydroxyacetone pathway. J. Biol. Chem. 272: $5544-5554$

90 Cabezas A., Costas M. J., Pinto R. M., Couto A. and Cameselle J. C. (2005) Identification of human and rat FAD-AMP lyase (cyclic FMN forming) as ATP-dependent dihydroxyacetone kinases. Biochem Biophys. Res. Commun. 338: 1682-1689

91 Andres S., Wiezer A., Bendfeldt H., Waschkowitz T., ToecheMittler C. and Daniel R. (2004) Insights into the genome of the enteric bacterium Escherichia blattae: cobalamin (B12) biosynthesis, B12-dependent reactions and inactivation of the gene region encoding B12-dependent glycerol dehydratase by a new mu-like prophage. J. Mol. Microbiol. Biotechnol. 8: 150-168

92 Huang S. H., Chen Y. H., Kong G., Chen S. H., Besemer J., Borodovsky M. et al. (2001) A novel genetic island of meningitic Escherichia coli $\mathrm{K} 1$ containing the ibeA invasion gene (GimA): functional annotation and carbon-source-regulated invasion of human brain microvascular endothelial cells. Funct. Integr. Genomics 1: 312-322

93 Corpet F. (1988) Multiple sequence alignment with hierarchical clustering. Nucleic Acids Res. 16: 10881-10890

94 Iushmanov S. V. and Chumakov K. M. (1988) [Algorithms for constructing phylogenetic trees of maximum topological similarity]. Mol. Gen. Mikrobiol. Virusol. 9-15 